\title{
Gleichstellung als arbeitspolitisches Feld. Symbolische Gewalt und Leistungsregime
}

Gleichstellungspolitiken scheinen an Relevanz gewonnen zu haben. Und zwar nicht nur auf der Ebene der Gesetzgebung. Auch Unternehmen können sich diesem Thema nicht mehr entziehen. Heißt das im Umkehrschluss, dass klassische Themen der Geschlechterforschung an Bedeutung verlieren und Analysen zu Ungleichheitsaspekten zwischen Frauen und Männern überflüssig werden? Am Beispiel von Pharma- und BiotechnologieUnternehmen gehen wir der Frage nach, ob sich gegenwärtig tatsächlich ein tief greifender Wandel der Arbeits- und Geschlechterpolitik abzeichnet oder lediglich neue „Egalitätsmythen“ geschaffen und Ungleichheiten somit de-thematisiert werden. Untersucht wird, ob das in wissensbasierten Branchen vorherrschende individualisierte Leistungsregime zur Verkennung von Geschlechterungleichheiten führt.

MARIA FUNDER, MARTINA SPROLL

\section{Einleitung}

Die Gleichstellungspolitik hat erkennbare Fortschritte erzielt. Dies spiegeln allemal die wachsende Anzahl von gesetzlichen Regelungen und Vereinbarungen - national sowie auf der europäischen Ebene - wider. Gibt es somit überhaupt noch einen Grund, sich weiterhin mit dem Thema Geschlechterungleichheit und Gleichstellung auseinanderzusetzen? Betrachtet man das Feld der Wirtschaft, dann könnte die Antwort auf den ersten Blick durchaus „nein“ lauten. Denn gerade in der Wirtschaft wird „Frauenförderung“ in der letzten Zeit zum aktiven Handlungsfeld. Wurde „Chancengleichheit" vor einigen Jahren noch stark moralisch begründet und war in erster Linie ein Aufgabenfeld von „Frauenbeauftragten“, dem kaum Beachtung geschenkt wurde, wird Geschlechtergerechtigkeit seit einiger Zeit verstärkt „im Rahmen eines ,Wirtschaftlichkeitsdiskurses' thematisiert" (Boes et al. 2013, S. 13), dem sich auch Gewerkschaften nicht entziehen. Ein besonders prominentes Beispiel ist die Telekom, die bis 201530 \% der Führungspositionen im oberen und mittleren Management mit Frauen besetzen will. Unternehmen können heutzutage eigentlich gar nicht mehr darauf verzichten, Maßnahmen zur Karriereförderung sowie zur Vereinbarkeit von Privat- und Berufsleben anzubieten, wenn sie nicht an gesellschaftlicher Legitimation verlieren wollen. Doch tragen solche Ansätze tatsächlich zu einer nachhaltigen Durchsetzung von Gleichstellung bei?
Eine zentrale These unseres Beitrages lautet: Viele Vereinbarkeitsmaßnahmen laufen letztlich gerade nicht auf Gleichstellung, sondern auf die Stabilisierung einer konservativen Familienpolitik hinaus. Die Erklärung hierfür findet sich in der gegenwärtigen Ausrichtung der Arbeitspolitik. Diese zeichnet sich zunehmend durch eine weitgehende Entgrenzung von Arbeitszeiten sowie durch ein individualisiertes Leistungsregime aus, das suggeriert: Es herrscht Geschlechtergerechtigkeit vor, denn worauf es ankommt ist Leistung, das Geschlecht spielt demnach keine Rolle. Wie sich im Zuge eines solchen Arbeitszeit- und Leistungsregimes und unter dem Mantel weit verbreiteter „Egalitätsmythen“(Funder 2014a) altbekannte Muster der Geschlechterungleichheit reproduzieren, insbesondere geschlechtliche Segregationen und der Gender Pay Gap, also das geschlechtsspezifische Lohngefälle zwischen Frauen und Männern, wollen wir in diesem Beitrag analysieren.

Argumentiert wird in zwei Schritten: Zunächst wird ein theoretisches Konzept vorgestellt, in dem wir einen umfassenden Begriff von Arbeits- und Geschlechterpolitik entwickeln, was sich auf Überlegungen von Burawoy und Bourdieu stützt (Abschnitt 2). In einem weiteren Schritt werden mit dieser theoretischen Perspektive zentrale Dimensionen der Arbeits- und Geschlechterpolitik im Hinblick auf die Branche, die Vereinbarkeitspolitik, die Leistungs- und Lohnpolitik sowie die Interessenvertretung rekonstruiert und näher beleuchtet (3). Dabei beziehen wir uns auf Ergebnisse unserer empirischen Studie 
zur Pharma- und Biotechtechnologie-Industrie. ${ }^{1}$ Schlussfolgerungen werden im letzten Teil gezogen (4).

\section{Arbeits- und Geschlechterpolitik als theoretisches Konzept}

Unsere These, dass Gleichstellung nur im Rahmen eines umfassenderen arbeitspolitischen Konzepts verstanden und angestrebt werden kann, erfordert einen neuen theoretischen Zugang. Wir schlagen daher ein um die Dimension „Geschlecht" erweitertes Verständnis von Arbeitspolitik vor, das darauf abzielt, nicht nur geschlechtsspezifischen Aushandlungsprozessen im Betrieb, sondern auch außerbetrieblichen Konstellationen bzw. Wechselwirkungen Rechnung zu tragen (vgl. Funder/Sproll 2012). Angesetzt wird auf drei Ebenen: Erstens muss die komplexe Verflechtung zwischen Erwerbs- und Reproduktionssphäre berücksichtigt werden, insbesondere die geschlechtshierarchische Arbeitsteilung in privaten Haushalten und das gesellschaftlich vorherrschende „Genderregime“ (vgl. u. a. Connell 1987). Zweitens geht es um die spezifischen Bedingungen und Machtverhältnisse der jeweiligen Branche und, drittens, um die Bedeutung des Arbeitsprozesses selbst in seiner sozialen und damit auch geschlechtlichen Form (z. B. die Frage der Segregation, Lohn- und Leistungspolitik, Macht- und Kontrollformen). Für ein solches Konzept greifen wir einen der wenigen theoretisch fundierten arbeitspolitischen Ansätze auf: die "politics of production“ des US-amerikanischen Soziologen Michael Burawoy (1985). Er konzipierte eine „politische Ökonomie des Arbeitsprozesses“, die den Betrieb als einen eigenständigen politischen Raum begreift, in dem sich soziale Beziehungen entlang des Arbeitsprozesses institutionalisieren. Kern seines Konzepts ist die Unterscheidung zwischen den „relations in production“ und den „relations of production“. Mit Ersteren richtet sich der Blick auf die betrieblichen Prozesse, also die sozialen Verhältnisse und Aushandlungsprozesse im betrieblichen Raum, die keineswegs "nur“ technisch determiniert sind. Mit den „relations of production" gelingt es ihm zudem, auch die außerbetrieblichen politischen, sozialen und ökonomischen Konstellationen sowie die staatliche Politik, die Kräfteverhältnisse auf (nationalen und internationalen) Märkten und die Reproduktionsweise von Arbeitskraft einzubeziehen. Somit ist Arbeitspolitik nicht auf den Betrieb beschränkt, sondern als Wechselwirkung unterschiedlicher Politiken zu verstehen, die in der „Arena“ Betrieb in spezifischer Weise konfiguriert und ausgehandelt werden.

Auch wenn Burawoy der Dimension „Geschlecht"kaum Bedeutung eingeräumt hat, bietet sein Ansatz wichtige Anschlussmöglichkeiten für eine geschlechterpolitische Erweiterung. In die Konzeption des Arbeitsprozesses als soziale Beziehung („relations in production“) schließen wir daher das Geschlechterverhältnis als eine grundlegende strukturierende Kategorie ein. Die „gender relations in production“ äußern sich z. B. in Form geschlechtlicher Segregationen und darunter liegenden stereotypisierenden $\mathrm{Zu}$ schreibungen sowie in der Persistenz des Gender Pay Gaps. Die ebenfalls zentrale Dimension der „relations of production" ermöglicht darüber hinaus auch, die komplexe Wechselwirkung zwischen Erwerbs- und Reproduktionssphäre (insbesondere der Haus- und Sorgearbeit) konzeptionell zu integrieren („gender relations of (re)production“) und damit die hiermit eng verwobene geschlechtsspezifische soziale Strukturierung und Dynamik, die bis heute immer noch vielfach durch eine geschlechtshierarchische Arbeitsteilung geprägt ist, zu erfassen. ${ }^{2}$ Doch reicht das aus, um die subtilen Prozesse von Macht und Herrschaft zu verstehen, die trotz des Anspruchs formaler Gleichbehandlung von Männern und Frauen immer wieder alte und neue Formen der Ungleichheit produzieren? Die feministische Forschung hat gezeigt, welch große Bedeutung symbolischen Repräsentationsformen und der Konstruktion von Körpern und Identitäten für eine vergeschlechtlichte soziale Praxis in Organisationen zukommt (siehe z. B. Acker 1990). Es bedarf folglich einer weiteren Öffnung unseres arbeits- und geschlechterpolitischen Konzepts, um die strukturelle mit der symbolischen Ebene zu verknüpfen. Wir greifen dafür auf grundlegende Kategorien aus Bourdieus Sozialtheorie zurück - insbesondere „Feld“, „Kapital“, „Habitus“, „Illusio“ und „symbolische Gewalt“ (vgl. hierzu ausführlicher Funder/Sproll 2012).

Der Bourdieu'sche Feldbegriff, angewendet sowohl auf die Branche als auch auf den Betrieb als relativ autonomes Teilfeld, ermöglicht einen Blick auf die jeweils spezifischen Konfigurationen und Regeln (Nomos), Herrschaftsverhältnisse, Legitimationsformen und Repräsentationen. Sie werden durch soziale Praxen, d. h. durch Kämpfe um Machtpositionen im Feld hergestellt, wobei unterschiedliche Akteure, je nach Geschlecht, Position, Bildungshintergrund, Klassenzugehörigkeit etc., über ein jeweils unterschiedliches ökonomisches, kulturelles, soziales und symbolisches Kapital verfügen. Das Teilfeld der Branche wird somit durch Akteure und Institutionen, wie Unternehmen, Lobbygruppen, Finanz- und Arbeitsmärkte (die wiederum durch spezifische Qualifikationsprofile und geschlechtliche Segregationen geprägt sind) bestimmt und weist zudem eine spezifische Arbeitsteilung auf (z. B. transnationale Zuliefernetzwerke). Es lässt sich folglich den „relations of produc-

1 Die von der Hans-Böckler-Stiftung finanzierte Studie wurde zwischen 2008 und 2011 an der Philipps Universität Marburg durchgeführt und basiert auf qualitativen Fallstudien in zwölf Pharma- und Biotechbetrieben in Deutschland (vgl. Funder/Sproll 2012).

2 Siehe hierzu auch die Debatte über den "male bias" in der Arbeitsforschung; vgl. u. a. Aulenbacher et al. 2007; Andresen/Völker 2005. 
tion“ im Burawoyschen Sinn zuordnen. Hier geht es um die politische Regulation der Branchenpolitik, u.a. staatliche Industrie- und Technologiepolitik, Gender-MainstreamingVorgaben der Europäischen Union (EU) sowie die Verbände- und Tarifpolitik. Auch die Konzeption des Betriebs als relativ autonomes Teilfeld (analog zu den „relations in production") erlaubt einen differenzierteren Blick auf die Positionen und Ressourcen der einzelnen Akteure und Personengruppen als bei Burawoy angelegt. Neben der (geschlechtsspezifisch) unterschiedlichen Ausstattung mit Macht liefert Bourdieu mit seinem Habituskonzept außerdem einen Erklärungsansatz für spezifische Geschlechterdispositionen und mit dem Begriff der „symbolischen Gewalt“ darüber hinaus Aufschluss über die „Verkennung“ und „Verschleierung“ von Geschlechterungleichheiten (vgl. u. a. Bourdieu 2005). Bourdieu zufolge ist der Habitus nicht angeboren, sondern in der sozialen Praxis erworben. Nicht nur Klassen- oder Schichtzugehörigkeit, sondern auch Zweigeschlechtlichkeit sind in ihn eingeschrieben. Im Zusammenspiel von Habitus und symbolischer Gewalt wird die Reproduktion und Persistenz von Geschlechterhierarchien sichtbar: Das zweigeschlechtliche Ordnungsschema ist sowohl bei Herrschenden als auch Beherrschten im Habitus verankert - die Ausübung von Herrschaft erscheint somit nicht unbedingt als Zwangsverhältnis, sondern ist wesentlich subtiler, denn sie setzt eine Verinnerlichung, ja sogar Einverständnis von beiden Seiten voraus. Dieses Einverständnis ist daher auch nicht als bewusster Akt zu verstehen, denn symbolische Gewalt wird nicht als Gewalt erkannt. Sie ist subtil, unsichtbar und äußert sich verdeckt, etwa durch Blicke, Gesten, Bemerkungen, Körperhaltungen, Intonation der Stimme etc. Diese Unsichtbarkeit bzw. Verschleierung von Herrschaftsbeziehungen (der "Spielregeln“) in einem Feld beschreibt Bourdieu mit dem Begriff der „Illusio“. Diese Spielregeln werden demnach ebenfalls von allen Akteurinnen und Akteuren im Feld als legitim anerkannt, verinnerlicht und können überhaupt nur so wirkungsmächtig werden. Dass sich mit diesem theoretischen Werkzeugkasten neue Einsichten zum Thema Arbeits- und Geschlechterpolitik gewinnen lassen, zeigt unsere Studie über die Pharma- und Biotech-Industrie.

\section{Arbeits- und Geschlechterpolitik in der Pharma- und Biotechnologie- Branche}

\subsection{Die geschlechtliche Dimension des Branchenfeldes (Relations of Production)}

Vor allem zwei Gründe haben uns bewogen, das Feld der Pharma- und Biotech-Industrie für unsere Studie auszuwählen:
(1) Die Zuschreibung als wissensintensive Industrie, die als Schrittmacherin von Innovation gilt und dementsprechend im Fokus staatlicher Technologieförderung steht; dies trifft insbesondere auf die noch junge, als „Start-up-Kultur“ entstandene Biotech-Branche zu.

(2) Die Besonderheit eines vergleichsweise hohen Anteils (hoch-)qualifizierter Frauen, die in der Pharmaindustrie traditionell in den Laborantinnenberufen und in der Biotechbranche unter den (Natur-)Wissenschaftlerinnen zu finden sind.

Uns bewegte u. a. die Frage, ob es in solchen hoch qualifizierten Bereichen eher zu einer Angleichung von Positionen kommt oder ob sich auch hier traditionelle Geschlechterhierarchien durchsetzen bzw. reproduzieren. Bevor wir dies jedoch mit Blick auf die betriebliche Arbeitspolitik diskutieren, gehen wir zunächst auf die spezifische Arbeitsteilung zwischen der Pharma-Industrie und den Biotech-Unternehmen ein, die sich erheblich auf die Geschlechterverhältnisse auswirkt. Wir begreifen die Pharma- und Biotech-Industrie als jeweils autonome Teilfelder (im Sinne des Bourdieu'schen Feldbegriffs), die jeweils ihre eigene Geschichte, politische und ökonomische Bedingungen und Machtverhältnisse entwickeln und durch unterschiedliche Akteure geprägt werden. Sie bilden jedoch auch ein gemeinsames Feld, da sie auf eine spezifische Weise miteinander verzahnt sind. Den Hintergrund dafür bildet die langjährige Problematik schwindender Produktivität und Innovationsfähigkeit der Pharmaunternehmen, während die in der Vergangenheit erworbenen Patente zunehmend auslaufen. Weltweit kam seit den 1990er Jahren ein tief greifender Restrukturierungsprozess in Gang, der nicht nur zu einer grundlegenden Neuordnung und Konzentration der Chemie- und Pharmaindustrie geführt hat, sondern auch eine wesentliche technologische Neuorientierung mit sich brachte: die Umstellung und Fokussierung auf biotechnologische Verfahren. „Frisches Know-how“ wurde vor allem von den jungen, dynamischen Biotech-Unternehmen eingebracht, die bis heute für die großen Pharmakonzerne die Rolle des Risikopuffers für kostenträchtige Innovationen übernehmen und als Gegenleistung oftmals auf strategische Kooperationen mit Pharmakonzernen zählen können. Hier begegnen sich allerdings keine gleichberechtigten Partner, sondern das neue Machtgefüge der Branche zeichnet sich durch ein asymmetrisches Verhältnis der beiden Branchensegmente aus: Die Biotech-Unternehmen sind in einer abhängigen und ökonomisch prekären Position gegenüber den großen Pharmakonzernen und haben nicht nur in Bezug auf ökonomisches Kapital „schlechte Karten“ im Konkurrenzkampf mit diesen. Dies spiegelt sich nicht zuletzt in einem großen Gefälle hinsichtlich der Beschäftigungssicherheit wider, denn viele Biotech-Unternehmen konnten sich nicht dauerhaft am Markt etablieren und mussten Konkurs anmelden. Hinzu kommt die Diskrepanz im Hinblick auf die Höhe der Gehälter sowie tarifliche und betriebliche Sozialleistungen, die in der Pharma-Industrie traditionell sehr 
umfangreich sind. Dieses Gefälle erzeugt einen Geschlechtereffekt: Positiv hervorzuheben ist zunächst, dass die Entstehung und Etablierung der Biotech-Branche in Deutschland eine hohe Nachfrage an qualifizierten Arbeitskräften mit sich gebracht hat. Insbesondere Frauen, deren Präsenz sich in Studienfächern wie Biologie, Biotechnologie und Molekularchemie in den letzten Jahren deutlich erhöht hat, erhielten so Zugang zu einem neuen und qualifizierten Arbeitsfeld. Dies erklärt den, verglichen mit der Pharma-Industrie, deutlich höheren Anteil von Frauen in wissenschaftlichen Positionen (Forschung und Entwicklung) der Biotech-Unternehmen (ca. 50-66\% in unseren Fallbetrieben). Im Kampf um hoch qualifizierte Arbeitskräfte scheinen Frauen für BiotechUnternehmen unverzichtbar geworden zu sein. Es gibt aber auch eine Kehrseite, nämlich die vergleichsweise schlechteren Beschäftigungsbedingungen in den Biotech-Unternehmen. Frauen sind offenbar eher bereit (oder gezwungen), die deutlich schlechter bezahlten und unsicheren Arbeitsplätze in der Biotech-Industrie zu akzeptieren. Und so führt die asymmetrische Arbeitsteilung zwischen Pharma- und Biotech-Industrie letztlich zu einer geschlechtsspezifischen Segregation, sodass sich trotz zunehmender Integration von Frauen auch in hoch qualifizierten Bereichen Geschlechterungleichheit reproduziert.

Legitimiert werden solche Asymmetrien in der betrieblichen Praxis durch die spezifischen Spielregeln des Feldes und eine entsprechende Illusio, die auch die (geschlechtsspezifischen) Strategien und Positionierungen der Beschäftigten prägen. So gilt die Pharma-Industrie als vergleichsweise hierarchisch, verkrustet und stärker von patriarchalen Strukturen geprägt; die Biotech-Industrie dagegen als weniger formal, stärker teamorientiert und persönlicher. In Interviews betonten vor allem Frauen, dass ihnen ein inhaltlich spannendes und persönlich angenehmes Arbeitsumfeld, für das die Biotech-Industrie steht, wichtig sei und dieses die vergleichsweise schlechten materiellen Bedingungen kompensiere.

Wirft man zudem einen Blick auf die Bedeutung staatlicher Regulation der Branche, fällt auf, dass viele der noch recht jungen Biotech-Unternehmen öffentliche Gelder sowohl aus der deutschen Technologieförderung wie auch aus europäischen Programmen erhalten haben. Letztere unterliegen, wie andere Förderprogramme, den Richtlinien des Gender Mainstreaming. Ein Vertreter der Dechema (Deutsche Gesellschaft für Chemische Technik und Biotechnologie e.V.) teilte uns mit, dass dieses Kriterium jedoch in der Praxis überhaupt keine Rolle spiele. Formal müsse den Anforderungen zwar genüge getan werden, dafür gebe es auch entsprechende Textbausteine für die Förderanträge, aber die Einhaltung werde von der EU weder eingefordert noch kontrolliert. Hinzu kommt - so seine Erfahrung -, dass die Anforderungen für die Innovationsförderung in der Biotechnologie oftmals mit traditioneller tariflicher und betrieblicher Regulation kollidiere, etwa wenn Langzeitversuche im Labor zu entgrenzten Arbeitszeiten führen, sodass in der Regel „Ärger“ mit Betriebsräten vorprogrammiert sei. Offenbar setzt die staatliche Förderung auf „Pioniergeist“, der dafür sorgt, dass mit „dem wenigen Geld und dem Idealismus der Leute" neue Produkte, auch um den Preis der Selbstausbeutung, vorangetrieben werden (Interview Dechema-Vertreter). Die Ausrichtung der deutschen Technologieförderung im Bereich Biotechnologie trägt somit zu einer Deregulierung und Prekarisierung bei, die sich letztlich in der oben beschriebenen Geschlechterdynamik äußert. Dies belegt sehr klar die Bedeutung der „relations of production", hier bezogen auf die Struktur und Regulation des Branchenfeldes für die Ausformung von Arbeitspolitik und der darin eingelassenen geschlechtsspezifischen Dynamik. Es zeigt auch, dass Gleichstellungspolitik offenbar viel tiefer und grundlegender ansetzen muss, als es GenderMainstreaming-Programme ermöglichen.

\subsection{Familienpolitik statt Gleichstellung: Vereinbarkeit als Schnittstelle zwischen „Relations in and of Production“}

Über Gleichstellung, insbesondere die Verankerung verbindlicher Regelungen (z. B. Frauenquoten), wird nun schon seit Jahren gestritten. Eine Vielzahl von Programmen und medienwirksamen Initiativen zu „Frau und Beruf“ wurde auf den Weg gebracht, zumal gerade in der Vereinbarkeitsproblematik nach wie vor ein „Karrierekiller“ für Frauen gesehen wird, der zu einem Verlust von „Humanressourcen“ führt. Die Pharmaindustrie ist schon deshalb ein hochinteressantes Forschungsfeld, weil sowohl auf der Branchen- als auch auf der betrieblichen Ebene große Anstrengungen zur Förderung der Vereinbarkeit von Beruf und Familie unternommen wurden. So wurde 2006 die Vereinbarung „Für eine chancengleiche und familienbewusste Personalpolitik ${ }^{\text {“ }}$ zwischen dem Arbeitgeberverband BAVC und der Gewerkschaft IG BCE abgeschlossen (BAVG/IGBCE 2006). Darin wird zwar auf die Verantwortung des Gesetzgebers, Rahmenregelungen zu schaffen, verwiesen, aber letztlich nur ein Appell an die Unternehmen gerichtet, dies durch betriebliche Maßnahmen zu begleiten. Formal verpflichtende Vorgaben, wie z. B. Quoten, werden zurückgewiesen, dagegen mögliche positive Effekte von Vereinbarkeitsmaßnahmen auf demografische Entwicklungen und Fachkräftemangel betont.

Diese Linie spiegelt sich in der betrieblichen Realität wider. Vor allem in den großen Pharma-Unternehmen gibt es eine Vielzahl von Vereinbarungen zu "Familie und Beruf", die geradezu als vorbildlich gelten können. Sie reichen von vielfältigen Teilzeitregelungen über Möglichkeiten der Kinderbetreuung bis hin zu Notfallnestern und Haushaltsdiensten (Mitnahme-Essen, Bügelservice usw.). Die Programme wurden in den meisten Unternehmen zertifiziert, wobei das Audit „Beruf und Familie“ der Hertie-Stiftung eine wichtige Rolle spielt. Zertifizierungen bieten eine gute Möglichkeit, sich öffentlich zu legitimieren, denn der Handlungsdruck für Unternehmen, Gleichstellungspolitik zu betreiben und vor allem „familienfreundlich“ zu sein, ist durch den 
gesellschaftlichen Diskurs immer größer geworden. Zertifikate bedeuten daher für Unternehmen einen Gewinn an symbolischem Kapital, wovon sie sich zudem Vorteile für die Rekrutierung qualifizierter Arbeitskräfte versprechen. So ist „der soziale Aspekt (zu einem) Standortfaktor geworden“, wie es im Interview mit einer Vertreterin der Personalabteilung eines unserer Pharma-Unternehmen formuliert wurde.

Auf den ersten Blick deutet offenbar alles darauf hin, dass viel für eine verbesserte Vereinbarungspolitik und damit auch - so der Grundtenor - für die Gleichstellung getan wird. Sieht man sich die Ausrichtung der betrieblichen Geschlechterpolitik jedoch genauer an, fällt auf, dass sie sich weitgehend auf das Thema „Vereinbarkeit“ beschränkt und keine bzw. nur wenige Maßnahmen direkt auf Gleichstellung zielen. Insbesondere die konkrete Förderung von Frauen in Führungspositionen erweist sich bislang als recht defizitär, vielfach gibt es sie auch gar nicht. Nicht selten findet sich in den Interviews die Formulierung, Frauen seien selbst schuld, denn sie setzten einfach „falsche“ Prioritäten, nämlich „Familie und Kinder“, anstatt eine berufliche Karriere anzustreben. Unsere Untersuchung zeigt dagegen, dass der Erfolg von Vereinbarkeit im Hinblick auf Gleichstellung vor allem durch ein arbeitspolitisches Regime infrage gestellt wird, das sich durch stark entgrenzte Arbeitszeiten auszeichnet. Erwartet und mitunter auch verlangt wird eine weitgehende Verfügbarkeit der Beschäftigten, was im Kern dem traditionellen männlichen Erwerbsarbeitsmodell entspricht, zeitlich sogar darüber hinaus geht und einer Vereinbarkeit von Beruf und Familie konträr gegenübersteht. Vereinbarkeit gelingt deshalb in der Regel nur unter Rückgriff auf Teilzeitmodelle. Diese werden jedoch zu 90 \% von Frauen in Anspruch genommen, was u. a. durch die unterschiedliche Bezahlung von Männern und Frauen motiviert ist. Zieht man außerdem in Betracht, dass die Unternehmen der Teilzeitbeschäftigung von Führungskräften überwiegend ablehnend gegenüberstehen, denn schließlich könne man - so der Vorgesetzte einer unserer Befragten - „keine Teilzeitkraft als Vorgesetzte von einer Vollzeitkraft nehmen“ (Beschäftigte, BioXenon, Interview 3), dann erklärt dies auch die Persistenz der vertikalen Geschlechtersegregation. Hinzu kommt, dass „eine Auszeit ohnehin immer schlecht für die Karriere (ist)“ (Personalleiterin, BioCarbo, Interview 7). So schließt sich hier der altbekannte Teufelskreis: Der Versuch, Vereinbarkeit über Teilzeit zu realisieren, führt zum Karriereeinbruch und reproduziert geschlechtshierarchische Arbeits- und Lebensmodelle, in denen Männern nach wie vor eher die Rolle des Familienernährers und Frauen die Hauptlast der Kindererziehungs- und Pflegeverantwortung zukommt. Unter solchen Bedingungen führen Vereinbarkeitsmaßnahmen zu einer konservativen Familienpolitik, die traditionelle Geschlechterhierarchien eher stärkt und einer proaktiven Gleichstellungspolitik entgegensteht - so ein zentrales Ergebnis unserer Studie. In den Biotech-Unternehmen bestätigte sich übrigens die gleiche Dynamik. Zwar gab es hier viel Entgegenkommen für in- dividuelle Vereinbarkeitsregelungen (verbindliche betriebliche und tarifliche Regelungen sind allerdings in der Regel nicht vorhanden), aber es dominieren gleichwohl entgrenzte Arbeitszeiten.

\subsection{Leistungspolitik und symbolische Gewalt („Relations in and of Production“)}

Mit der Leistungspolitik adressieren wir ein weiteres Kernthema der Arbeitspolitik, das ebenso wie die Arbeitszeitpolitik hoch relevant für die große Beharrungskraft von Geschlechterungleichheiten ist. In den Diskussionen über Gleichstellung ist dieses Thema jedoch kaum präsent, im Gegenteil: Zumeist wird jegliche Art der Ungleichbehandlung von Männern und Frauen in der Leistungsbeurteilung negiert - und zwar sowohl seitens der Unternehmen als auch der Beschäftigten. Das Credo „moderner“ Arbeits- und Geschlechterpolitik, das sich - wie bereits ausgeführt - branchenübergreifend verallgemeinert hat, lautet: Nicht Geschlecht, sondern individuelle Leistung zählt! Damit wird jede Art der Gleichstellungspolitik als „leistungsfeindlich“ und sogar diskriminierend de-legitimiert, obwohl gleichzeitig eklatante Unterschiede in der Bezahlung von Männern und Frauen erkennbar sind, ganz abgesehen von einer weiterhin vorherrschenden geschlechtshierarchischen Arbeitsteilung, die vor allem in der bis heute anhaltenden Unterrepräsentanz von Frauen in gehobenen Positionen zum Ausdruck kommt. Hier greift offenbar ein Verkennungseffekt, der sich in einem „universalisierten Code der Gleichheit“" (Dölling 2004) bzw. in einem „Egalitätsmythos“ (Funder 2014a) ausdrückt. Erklärbar ist dieser Widerspruch durch das Zusammentreffen von strukturellen Gegebenheiten mit geschlechtsspezifischen habituellen Dispositionen und insbesondere symbolischer Gewalt.

\subsubsection{Die Illusio gerechter Leistung und die Ver- schleierung von Geschlechterungleichheit}

Die hoch qualifizierten Bereiche „Forschung und Entwicklung" der Pharma- und Biotech-Industrie sind durch eine projektförmige Arbeitsorganisation geprägt, die einen hohen persönlichen Einsatz der Beschäftigten sowie hohe Identifikation mit dem Produkt und - vor allem in der durch eine „Start-up-Kultur“ geprägten Biotech-Branche - auch mit dem Unternehmen erfordern. Die Ausrichtung des Leistungsregimes bei den Biotech-Unternehmen lässt sich auf folgenden Nenner bringen: „Pay for Performance, not for Presence“. Damit ist gleichzeitig ein Kontrollregime benannt, das auf Vertrauensarbeitszeit beruht. Ein massiver Trend zur Entgrenzung von Arbeitszeiten ist die Folge, der in der Pharma-Industrie nicht ganz so weitgehend, aber auch hier zu konstatieren ist - und dies, obwohl in den tariflichen Bereichen noch formale Arbeitszeitregelungen existieren, die dem eigentlich entgegenstehen. Damit korrespondiert ein individuell orientiertes Leistungsregime, das auf Zielvereinbarungen setzt, in denen persönliche 
und oftmals auch unternehmens- und abteilungsbezogene Leistungsziele formuliert werden. Insbesondere in den Biotech-Betrieben wird dabei der Betrieb als „Familie“ konstruiert, in der es um „Geben und Nehmen“, also das Prinzip der Gegenseitigkeit bzw. „reziproken Tausch“ geht. Hier spielen persönliche Beziehungen und Abhängigkeiten eine große Rolle. Die Beschäftigten werden so zu Höchstleistungen motiviert, die nicht nur die massive Entgrenzung von Arbeitszeiten zur Folge hat, sondern auch eine weitreichende Übertragung von Verantwortung für Innovation und Wissensproduktion, aber auch von unternehmerischem Risiko auf die Beschäftigten. Dies trifft sich mit einer habituellen Prägung von Naturwissenschaftlerinnen und -wissenschaftlern, deren Arbeitsmotivation aus der Sinnerfüllung durch Forschung und Innovation resultiert.

Diese Zusammenhänge formen die Basis für die spezifische „Illusio“ des Feldes, die im Kern darin besteht, dass erstens der Wunsch nach Selbstverwirklichung in der Arbeit mit einer ständigen Anrufung des „unternehmerischen Selbst“ (Bröckling 2007) verknüpft und dies zweitens mit dem Leitbild „gerechter"Leistungsnormen verbunden wird. Symbolische Gewalt drückt sich darin aus, dass diese Leistungsnormen in einem komplexen Zusammenspiel von Identifikation, persönlicher Motivation und Verkennung ihres inhärenten Zwangscharakters von Beschäftigten anerkannt und verinnerlicht werden - trotz enormer gesundheitlicher Belastungen (auffallend waren insbesondere psychische Erkrankungen und Burn-Out-Probleme). Die Vorstellung gerechter Leistungsnormen stützt sich auf die Annahme, dass jeder und jede nach seiner bzw. ihrer persönlichen Leistung beurteilt, bezahlt und mit Aufstiegsmöglichkeiten belohnt wird - ganz im Gegensatz etwa zu kollektiven Formen der Bezahlung, wie z. B. der Ausschüttung einer für alle gleich großen Gewinnbeteiligung. Letzteres wurde von Beschäftigten in unseren Interviews als ungerecht zurückgewiesen, da eine finanzielle Gleichbehandlung persönliche Leistungsanstrengungen negiere und dafür die „Faulen“ belohne. Eine auf individuelle Leistung bezogene Vorstellung von Gerechtigkeit beruht also geradezu auf konkurrierenden Praktiken der Distinktion, d. h. auf Hierarchiebildungen, die sozial akzeptiert und verinnerlicht sind (wie z. B. geschlechtsspezifische Dispositionen) und dadurch auch herrschaftswirksam werden können. Unser Argument ist, dass dadurch auch geschlechtliche Hierarchien neu geformt und gefestigt werden, obwohl die Illusio gerechter individueller Leistung einer solchen Vorstellung gerade entgegensteht. Zwar spielt z. B. im qualifizierten Bereich naturwissenschaftlicher Forschung das Geschlecht in der alltäglichen Teamarbeit auf den ersten Blick offenbar keine Rolle, d. h. zumindest auf der sachbezogenen Ebene scheint es durchaus ein „Vergessen von Geschlecht“ (Hirschauer 2001) zu geben. Schaut man aber genauer hin, dann zeigen sich am Ende doch wieder geschlechtsspezifische Klassifikationsmuster, die zum einen mit Geschlechterstereotypisierungen und zum anderen mit der bereits beschriebenen Illusio der (Hoch-)Leistung zu erklären sind.
Ein wichtiger Indikator hierfür ist der allseits präsente Diskurs über die überdurchschnittliche Leistungsbereitschaft von Frauen und ihren „Hang zum Perfektionismus“, der sich sowohl in Fremd- als auch in Selbstzuschreibungen äußert. Dies bedeutet offenbar, dass die Erfüllung der beschriebenen Leistungsnormen Frauen den Eindruck vermittelt, sie müssten einen höheren Einsatz zeigen, was sich z. B. in den folgenden Interviewaussagen bestätigt: „Ich glaube, der Druck kommt eigentlich mehr von mir selber, (einem Hang zur, d. V.) Perfektion [...]“ (Beschäftigte, BioXenon, Interview 2). „Ich glaube schon, dass es diese Belastung hauptsächlich der Frau ist, die irgendwo glaubt, sie muss jetzt besonders gut, besonders intensiv arbeiten" (Managerin, BioLithio, Interview 9b).

Erklärungen dafür liegen auf unterschiedlichen Ebenen: Erstens entspricht die wahrgenommene Anforderung der uneingeschränkten zeitlichen Verfügbarkeit einer männlichen Norm, der Männer mit Kindern nach wie vor eher entsprechen können als Frauen mit Kindern. Dies bringt Frauen dazu, dass sie diese Unterstellung durch besonderen Einsatz auszugleichen versuchen. Zweitens gelten auch Frauen ohne Kinder aufgrund potenzieller Gebärfähigkeit als ein „Risikofaktor“, der Personalentscheidungen und die Erwartung zukünftiger Leistungsfähigkeit negativ beeinflusst. Entsprechende Klassifikationsschemata und habituelle Ausprägungen äußern sich, drittens, in Vorbehalten gegenüber Frauen, wenn es um die Besetzung von Führungspositionen oder um die Entscheidung einer höheren Gehaltseinstufung geht - was nicht zuletzt zu den beträchtlichen Gehaltsunterschieden führt. Umgekehrt weisen Frauen und Männer mitunter auch ein unterschiedliches Selbstverständnis hinsichtlich ihrer Ansprüche auf Höherstufung und Beförderung auf. In den Interviews unterstrichen Frauen entsprechend solcher geschlechtsspezifischer Klassifikationsmuster oftmals, dass ihnen weniger an einem höherem Gehalt oder formalen Titeln liege als vielmehr an einer interessanten, zufriedenstellenden Arbeit. Zieht man außerdem in Betracht, dass das Leistungsregime, basierend auf individuellen Zielvereinbarungen, zu einer Re-Personalisierung der Leistungsbeurteilung durch Vorgesetzte beiträgt, gehen Beschäftigte, die jeweils mit unterschiedlichen habituellen und geschlechtsspezifischen Dispositionen ausgestattet sind, in Verhandlungen, in denen ein verschärfter Zwang zur Selbstvermarktung und zum „Impression Management" vorherrscht.

Hier äußern sich also verschiedene Mechanismen, die wir als eine subtile, auf symbolischer Gewalt beruhende Praxis der Machtausübung deuten. Sie tragen dazu bei, dass sich Geschlechtersegregationen und Verteilungsasymmetrien reproduzieren und nicht grundsätzlich infrage gestellt werden, zumal die scheinbar geschlechtsneutrale Subjektivierung durch ein individualisiertes Leistungsregime $\mathrm{zu}$ einer Organisationskultur führt, die einer aktiven Gleichstellungspolitik diametral entgegensteht (vgl. hierzu auch Andresen/Völker 2005). Dies zeigt sich auch in der weitreichenden De-Thematisierung und De-Legitimierung von 
Geschlecht bzw. geschlechtlicher Ungleichheit. Folgende Gleichheitscredos sind hierfür typisch: „[...] Bei uns ist vollkommen egal, ob man Mann oder Frau ist“ (Managerin, PharmaGamma, Interview 18) oder „[...] wir behandeln Frauen nicht als wären sie behindert und brauchen Extraförderung [...] “ (Managerin, PharmaDelta, Interview 13). $\mathrm{Da}$ es jedoch nach wie vor geschlechterdifferenzierende Zuschreibungen von Leistungsfähigkeit sowie eine geschlechtsspezifisch ungleiche Verteilung von sozialem und symbolischem Kapital gibt, was wiederum für die persönliche Machtposition und die geschlechtsspezifische Hierarchiebildung von entscheidender Bedeutung ist, kommen wir zu folgendem Schluss: Geschlechterklassifikationen werden gleichzeitig relevanter, widersprüchlicher und unsichtbarer. Sie sind angesichts der damit verbundenen $\mathrm{Ab}$ spaltungen, Tabuisierungen und Individualisierungen nur sehr schwer auszuhebeln.

\subsubsection{Aushandlungsprozesse: Die Rolle der Interessenvertretungen}

Ein zentraler Befund unserer Studie lautet: Eine weitreichende Institutionalisierung von Gleichstellungspolitik hat (bislang) weder in den untersuchten Biotech- noch in den Pharma-Unternehmen stattgefunden. Mit Blick auf die Pharma-Unternehmen liegt dies zum einen daran, dass sich Gleichstellungspolitik - wie beschrieben - in erster Linie auf weitgehend an Frauen adressierte Maßnahmen zur Vereinbarkeit von Beruf und Familie beschränkt, die aber nicht durch weitergehende betriebliche Gleichstellungsmaßnahmen flankiert werden und (noch) weit davon entfernt sind, auf einen Wandel von Geschlechterleitbildern - bei Männern und Frauen - hinzuwirken. Betriebliche Interessenvertretungen bilden in den von uns untersuchten Fallbetrieben kein Gegengewicht gegenüber der konstatierten De-Thematisierung und Negation geschlechtsspezifischer Ungleichheiten, die dazu führt, dass Forderungen nach Gleichstellungsmaßnahmen vielfach als rückwärtsgewandt und „Opferdiskurs“ angesehen werden, den moderne und selbstbewusste Frauen nicht nötig hätten. Eine proaktive Gleichstellungspolitik wird nicht als ein zentrales Handlungsfeld empfunden, sondern rangiert im Hinblick auf den Stellenwert bzw. die symbolische Bedeutung, die diesem Thema zugemessen wird, in den Gremien insgesamt weit unten. Zwar gibt es einzelne sehr engagierte Betriebsratsmitglieder, doch diese haben es angesichts solcher Hierarchisierungen schwer, ihre Position deutlich zu machen und Mehrheiten zu bilden. Betriebsräte werden zudem bei individuellen Aushandlungsprozessen, etwa bezüglich der Höhergruppierung oder der Vereinbarung von Arbeitszeitsowie Teilzeitregelungen, gar nicht mehr eingeschaltet und von einem Teil der Beschäftigten als nicht mehr zeitgemäß empfunden und ignoriert, vielfach sogar abgelehnt. Die deutliche Schwächung kollektiver Organisationsformen der Beschäftigten schränkt auch die Grundlagen und Bedingungen für Geschlechterpolitik ein, was allerdings keines- wegs bedeutet, dass Geschlechterfragen im umgekehrten Fall einer relativ strategiefähigen Interessenvertretung automatisch berücksichtigt würden.

Im Gegensatz zur Pharma-Industrie, in der Mitbestimmungspolitik nach wie vor eine Rolle spielt, handelt es sich bei der Biotech-Branche bis heute überwiegend um eine "gewerkschafts- und betriebsratsfreie Zone“, was sich zukünftig kaum ändern wird, wenn es Gewerkschaften nicht gelingt, neue strategische Konzepte zu entwickeln, die auf dieses Teilfeld zugeschnitten sind. Die Bilanz in den in ihrem Selbstverständnis modernen, gleichberechtigten und partizipativen Biotech-Unternehmen ist damit noch ernüchternder als in der eher konservativen Pharma-Industrie. Dass sich hieraus sowie aus der zunehmenden Flexibilisierung, Intensivierung und Entgrenzung von Arbeit weitreichende Konsequenzen für die Ausgestaltung der Arbeitspolitik ergeben, ist unstrittig; dass es dabei immer auch um Geschlechterpolitik geht, wird hingegen oftmals nicht weiter reflektiert.

\section{Schluss: Gleichstellung als arbeitspolitisches Handlungsfeld}

Am Beispiel der Pharma- und Biotech-Branche wird deutlich, wie dringend ein Wandel der betrieblichen, über- und außerbetrieblichen Arbeits- und Geschlechterpolitik ist. Im Kern unserer Argumentation steht das individualisierte Leistungsregime, das zum einen durch eine weitgehende Entgrenzung von Arbeitszeiten geprägt ist und Vereinbarkeit zumeist nur durch Teilzeitarbeit und damit auf Kosten von Aufstiegschancen ermöglicht, sodass sich letztlich traditionelle Muster geschlechtshierarchischer Arbeitsteilung verfestigen. Zum anderen führt es zu einer Individualisierung und Entsolidarisierung, was nicht nur zu einer massiven Intensivierung von Arbeit und Selbstausbeutung beiträgt, sondern unter dem Deckmantel der Gleichheit auch geschlechtsspezifische Hierarchien (im Zuge habitueller Dispositionen und symbolischer Gewalt) in widersprüchlicher Weise verstärkt und gleichzeitig unsichtbarer macht. Nimmt man einen solchen Befund ernst, dann kann Gleichstellungspolitik nur scheitern, wenn sie sich auf rein "additive“ Maßnahmen und Programme beschränkt - sie muss darüber hinaus an Kernthemen der Arbeitspolitik ansetzen. Das bedeutet aber nicht nur, dass Gewerkschaften und betriebliche Interessenvertretungen sich der Frage von Gleichstellung und Geschlechtergerechtigkeit in neuer Weise stellen müssten, sondern dass sie sich auch gegenüber Themen wie Arbeitszeit (z. B. in einer Lebensverlaufsperspektive), Leistungsbeurteilung, Bezahlung und Prekarisierung in anderer Weise positionieren müssten, um verlorenes und neues Terrain (wieder) zu gewinnen. Das kann nur gelingen, wenn Gleichstellungspolitik strategisch als ein inhärenter Bestandteil des „traditionellen Geschäfts“ 
betrieblicher und gewerkschaftlicher Arbeitspolitik wahrgenommen und weiterentwickelt wird (vgl. Funder 2014b).

Unsere Befunde zeigen, wie verwoben die vorherrschenden Geschlechterordnungen mit der Ausgestaltung von Arbeitspolitik nicht nur im betrieblichen, sondern auch im außerbetrieblichen Feld sind. Dabei kommen sowohl der Branchen-/Technologiepolitik und der Regulation des Arbeitsmarkts als auch - und das ist sicher nichts Neues - vor allem der Organisation der gesellschaftlichen Reproduktionsarbeit eine wichtige Rolle zu. Aller Rhetorik über Vereinbarkeit von Beruf und Familie und Work-Life-Balance zum Trotz spiegelt sich in unseren betrieblichen Fallstudien eine gesamtgesellschaftliche Entwicklung, die dem gerade entgegensteht und sich mit Begriffen wie Vermarktlichung oder Ökonomisierung, Beschleunigung, Flexibilisierung, Individualisierung und Leistungsorientierung beschreiben lässt. Andresen und Völker formulieren es folgendermaßen: „Es verallgemeinert sich die Anforderung, flexibel mehrere Lasten und Aufgaben gleichzeitig managen zu können, sich an betriebliche Erfordernisse anzupassen, immer sein Bestes zu geben, Positionen niemals für gesichert zu halten, ständig auf dem Sprung zu sein, etc.“ (Andresen/Völker 2005, S. 109). Damit wird jedoch gerade die Balance zugunsten der Produktionssphäre verschoben und die Verantwortung für die gesellschaftliche Organisation der Reproduktion immer stärker individualisiert. Dies führt zu einer Relativierung des außerbetrieblichen Lebens, während sich unter dem Deckmantel vorherrschender Normen, wie etwa dem Leistungsprinzip, und sogar des Diskurses über Work-Life-Balance symbolische Herrschaftsverhältnisse entwickeln, die auch, aber nicht nur, entlang von traditionellen geschlechterbezogenen Formen der Ungleichheit verlaufen. Bourdieu hat nicht umsonst den Begriff der symbolischen Gewalt verwendet, um genau auf solche „verborgenen Mechanismen der Macht“ hinzuweisen. Sich aktiv dagegen zu stellen, bedeutet, stärker zu reflektieren, wo auch Gewerkschaften und betriebliche Interessenvertretungen die beschriebenen Spielregeln bzw. die Illusio selbst mit reproduzieren oder sie auch konterkarieren könnten. Das wiederum erfordert, erst einmal die vorherrschende Illusio zu entschlüsseln und die eigentlichen Machtverhältnisse, die darunter liegen, zu erkennen. Und so können wir nur unterstreichen, was von Alemann (2007) unter Bezug auf Traute Hoffmann hervorhebt, nämlich dass es bei den Auseinandersetzungen um Gleichberechtigung um „Verteilungskämpfe“ geht. Institutionell verankerte Rechte und verbindliche Rahmenbedingungen haben damit keineswegs an Bedeutung verloren; im Gegenteil, gerade Geschlechterpolitik kommt ohne sie nicht aus, denn sonst wird sie - wie die Studie zeigt - schnell zu einer rein symbolischen Politik.

\section{LITERATUR}

Acker, J. (1990): Hierarchies, jobs, and bodies: A theory of gendered organizations, in: Gender \& Society 4 (2), S. 139-158

Alemann, A. von (2007): Gleichstellung per Gesetz? Vom Gesetzentwurf zum Deal zwischen Regierung und Wirtschaftsverbänden, in: Imbusch, P./Rucht, D. (Hrsg.): Profit oder Gemeinwohl? Fallstudien zur gesellschaftlichen Verantwortung von Wirtschaftseliten, Wiesbaden, S. 161-199

Andresen, S./Völker, S. (2005): Hat das Arbeitssubjekt der Zukunft (k)ein Geschlecht? Überlegungen zur Analyse der aktuellen Umbrüche in der Arbeit aus genderkritischer Perspektive, in: Lohr, K./Nickel, H. M. (Hrsg.): Subjektivierung von Arbeit. Riskante Chancen, Münster, S. 92-114 Aulenbacher, B./Funder, M./Jacobsen, H./Völker, S. (2007): Forschung im Dialog - Einleitung, in: Dies. (Hrsg.): Arbeit und Geschlecht im Umbruch der modernen Gesellschaft, Wiesbaden, S. 9-23

Boes, A./Bultemeier, A./Trinczek, R. (2013): Ein historischer Möglichkeitsraum für die Karrierechancen von Frauen - zur Einführung, in: Dies. (Hrsg.):
Karrierechancen von Frauen erfolgreich gestalten. Analysen, Strategien und Good Practices aus modernen Unternehmen, Wiesbaden, S. 13-34

Bourdieu, P. (2005): Die verborgenen Mechanismen der Macht enthüllen, in: Ders. (Hrsg.): Die verborgenen Mechanismen der Macht, Schriften zur Politik \& Kultur (1), Hamburg, S. 81-86

Bröckling, U. (2007): Das unternehmerische Selbst. Soziologie einer Subjektivierungsform, Frankfurt a. M.

Bundesarbeitgeberverband Chemie (BAVC)/Industriegewerkschaft Bergbau, Chemie, Energie (IG BCE) (2006): Chancengleichheit: Für eine chancengleiche und familienbewußte Personalpolitik. Gemeinsame Grundsätze des Bundesarbeitgeberverbandes Chemie und der IG Bergbau, Chemie, Energie, http://www.chemie-sozialpartner.de/vereinbarungen/chancengleichheit/ familien bewusste-personalpolitik/ (letzter Zugriff: 04.03.14)

Burawoy, M. (1985): The politics of production: Factory regimes under capitalism and socialism, London

Connell, R. (1987): Gender and power. Society, the person, and sexual politics, Stanford

Dölling, I. (2004): Männliche Herrschaft als paradigmatische Form der symbolischen Gewalt, in: Steinrücke, M. (Hrsg.): Pierre Bourdieu - Politisches Forschen, Denken und Eingreifen, Hamburg, S. 73-90

Funder, M. (2014a): Einführung: „Gender Cage - Revisited“, in: Dies. (Hrsg.): Gender cage - revisited. Handbuch zur Organisations- und Geschlechterforschung, Baden-Baden, S. 9-25

Funder, M. (2014b): Arbeitspolitik: Konzepte und Perspektiven, in: Wiechmann, E./Bogumil, J. (Hrsg.): Demokratie im Wandel, Baden-Baden, S. 59-83 Funder, M./Sproll, M. (2012): Symbolische Gewalt und Leistungsregime. Geschlechterungleichheit in der betrieblichen Arbeitspolitik, Münster Hirschauer, S. (2001): Das Vergessen des Geschlechts. Zur Praxeologie einer Kategorie sozialer Ordnung, in: Heintz, B. (Hrsg.): Geschlechtersoziologie, Kölner Zeitschrift für Soziologie und Sozialpsychologie (Sonderheft 41), Opladen, S. $208-235$

\section{AUTORINNEN}

MARIA FUNDER, Prof. Dr., lehrt an der Philipps-Universität Marburg Soziologie. Arbeitsschwerpunkte: Arbeits- und Wirtschaftssoziologie, Industrial-Relations, Organisations- und Geschlechterforschung.

funder@staff.uni-marburg.de

MARTINA SPROLL, Dr., ist Wissenschaftliche Mitarbeiterin im Bereich Soziologie des Lateinamerika-Instituts der Freien Universität (FU) Berlin. Arbeitsschwerpunkte:Transnationale Produktion, Industrie- und Arbeitssoziologie, Geschlechterverhältnisse, soziale Ungleichheit, Intersektionalität.

martina.sproll@fu-berlin.de 